\title{
Cardiovascular magnetic resonance features of caseous calcification of the mitral annulus Lorenzo Monti*1,2,3, Eva Renifilo $^{1}$, Manuel Profili ${ }^{1}$ and Luca Balzarini ${ }^{1}$
}

Address: ${ }^{1}$ Department of Radiology, I.R.C.C.S. Istituto Clinico Humanitas, Rozzano, Milan, Italy, ${ }^{2}$ Department of Cardiology, I.R.C.C.S. Istituto Clinico Humanitas, Rozzano, Milan, Italy and ${ }^{3}$ Cardiovascular Magnetic Resonance, I.R.C.C.S. Istituto Clinico Humanitas, Via Manzoni 56, 20089 Rozzano (MI), Italy

Email: Lorenzo Monti* - lorenzo.monti@humanitas.it; Eva Renifilo - evarenifilo@yahoo.it; Manuel Profili - manuel.profili@humanitas.it; Luca Balzarini - luca.balzarini@humanitas.it

* Corresponding author

\section{Published: 26 May 2008}

Journal of Cardiovascular Magnetic Resonance 2008, 10:25 doi:10.1 I86/I532-429X-1025

This article is available from: http://www.jcmr-online.com/content/I0/I/25

(C) 2008 Monti et al; licensee BioMed Central Ltd.

This is an Open Access article distributed under the terms of the Creative Commons Attribution License (http://creativecommons.org/licenses/by/2.0), which permits unrestricted use, distribution, and reproduction in any medium, provided the original work is properly cited.
Received: 3 April 2008

Accepted: 26 May 2008

\author{
Abstract \\ We present two cases of caseous calcification of the mitral annulus studied by Cardiovascular \\ Magnetic Resonance; the diagnostic feature of this rare cardiac mass are described.
}

\section{Introduction}

Cardiovascular Magnetic Resonance (CMR) is unrivalled as an imaging modality for the evaluation of cardiac and pericardial masses. Caseous calcification of the mitral annulus is a rare [1-4] form of degeneration of the fibrous skeleton of the mitral annulus that should be included in the differential diagnosis of myocardial masses. Usually found in elderly patients, it's typically located in the posterior mitral annulus. We studied with CMR two cases of caseous calcification of the mitral annulus; in both cases the diagnosis was confirmed with a CT scan.

\section{Case report}

Patient 1. A 87-year-old woman was referred to our Hospital with suspicion of an atrial mass. A CMR study (Figure 1) showed appearances compatible with extensive caseous calcification of the posterior mitral annulus, with dimensions of $3 \times 2.5 \mathrm{~cm}$ and a circumferential extension of about $5 \mathrm{~cm}$, in the basal inferior wall of the left ventricle and bulging into the posterior left atrium, without significant mitral valve regurgitation.
Patient 2. A 70-year-old male, who had undergone bone marrow transplantation for a follicular non-Hodgkin's lymphoma, was referred after transthoracic echocardiography had identified a hyperechogenic intramyocardial mass in the postero-lateral basal wall. CMR (Figure 2) and CT scan (Figure 3 ) confirmed the diagnosis of caseous calcification of the mitral annulus.

Both patients were asymptomatic at the time of diagnosis and were treated conservatively.

\section{CMR features}

The CMR findings of the two patients are similar. In T1weighted (T1W) sequences (Panel A in Fig. 1 and 2) the masses are dark, and in fat suppressed T2-weighted (T2W) STIR sequences (Panel B in Fig. 1 and 2) they lack signal. The combination of dark T1W and T2W tissue signal is unusual for a cardiac mass [5] and suggests calcification. In balanced steady state free precession (bSSFP) images the regions of caseous calcification ( ${ }^{*}$ in Panel C in Fig. 1 and 2) appear only slightly darker than the normal myocardium, with a well-defined intramyocardial border. During first pass gadolinium contrast administration no 


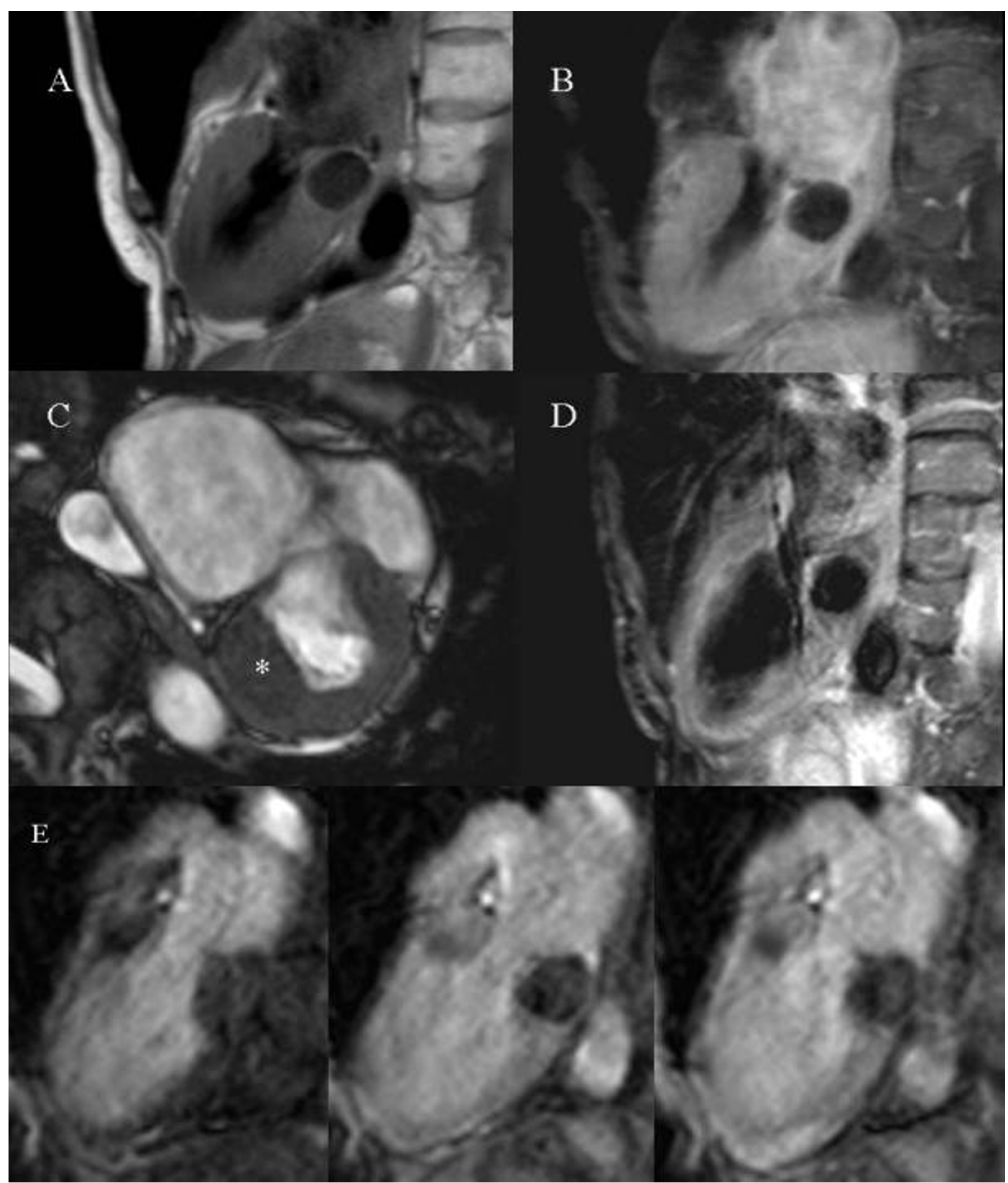

\section{Figure I}

Patient I: Panel A: TI-W turbo spin echo sequence. Panel B: STIR (Short Tau Inversion Recovery) sequence. Panel C: bSSFP sequence. Panel D: post-contrast TI-W turbo spin echo sequence. Panel E: first pass perfusion. 


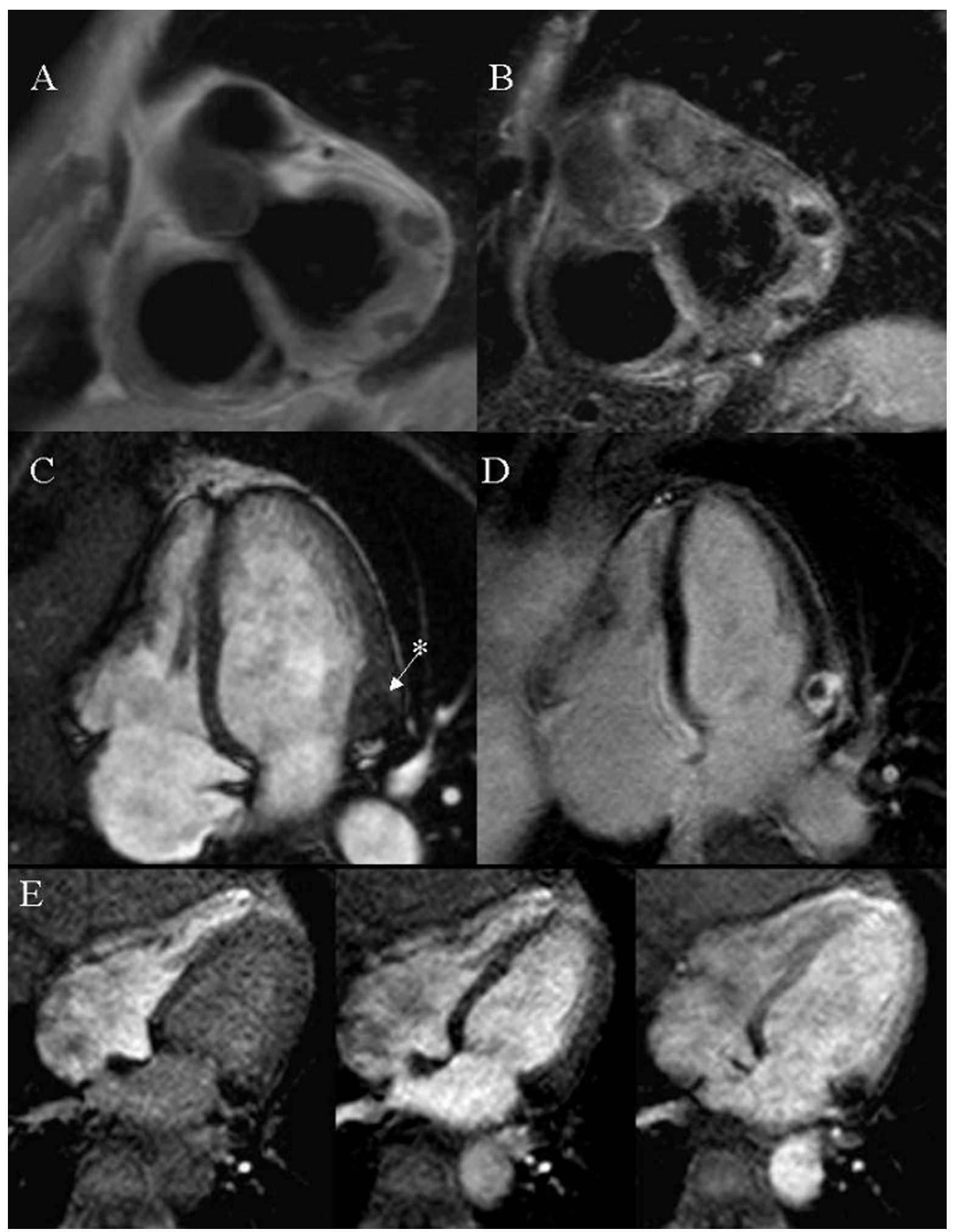

\section{Figure 2}

Patient 2: Panel A: TI-W turbo spin echo sequence. Panel B: STIR (Short Tau Inversion Recovery) sequence. Panel C: bSSFP sequence. Panel D: Delayed Enhancement, 10 minutes after contrast administration: an enhanced rim appears to surround a non-enhanced core. Panel E: first pass perfusion. 


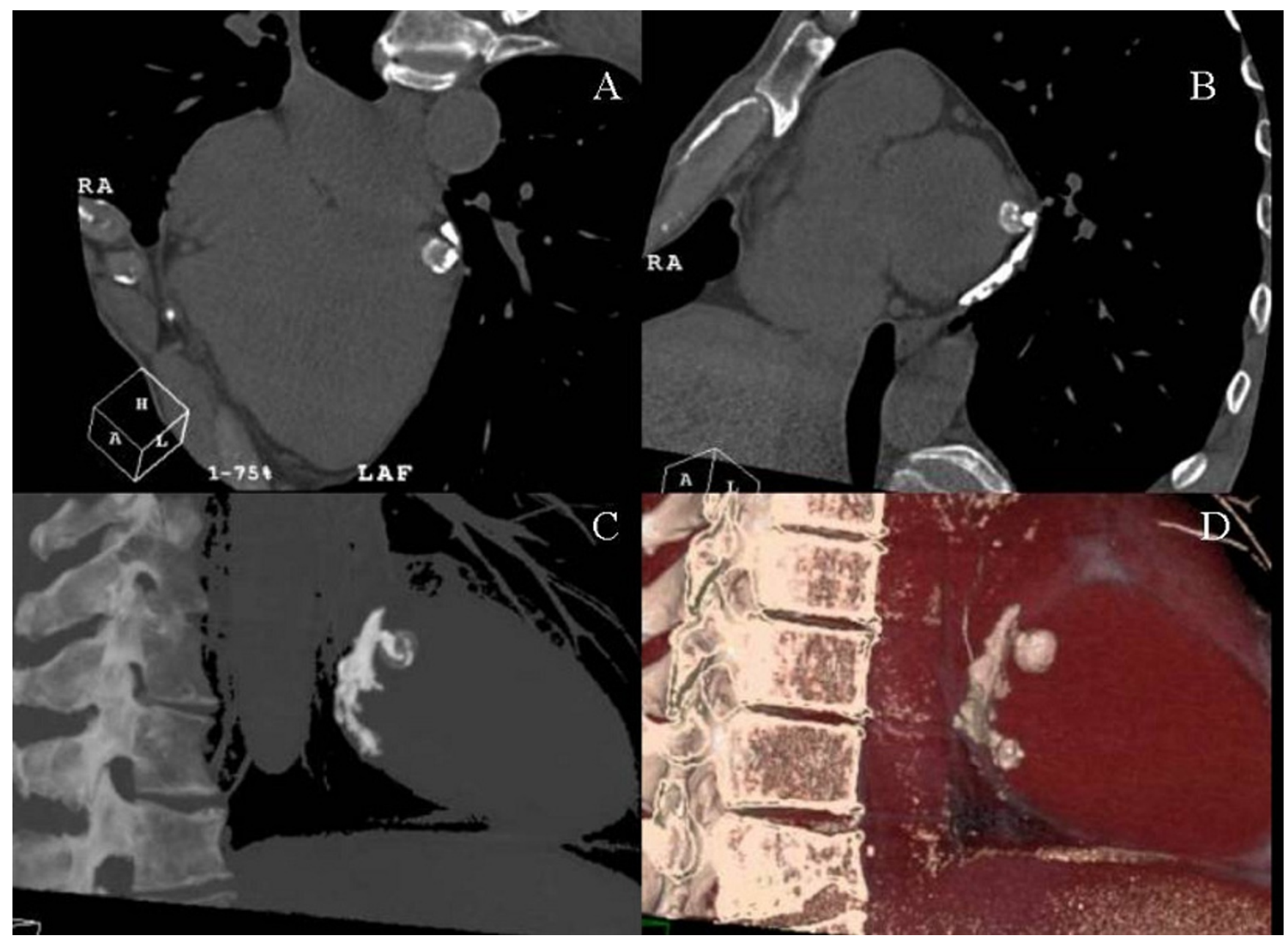

Figure 3

Patient 2: ECG-triggered 64-slices CT scan. Volume rendering images. Panel A: horizontal long axis. Panel B: short axis at the mitral annulus level. Panel C and D: the caseous calcification involves the postero-lateral mitral annulus and shows inhomogeneous attenuation.

enhancement can be observed (Panel E in Fig. 1 and 2). There was evidence of perfusion delay in the anterior mitral annulus (Fig. 1) and in the septal mitral annulus (Fig. 2), suggesting local extension of the disease process. Post-contrast T1-weighted sequence (Panel D in Fig. 1) is negative for enhancement of the mass, but fibrous tissue seems to surround and delimitate the caseous core. Delayed enhancement sequences were obtained only in patient 2 (Panel D in Fig. 2); an enhanced fibrous cap was found to surround a central core that showed no contrast enhancement.

Apart from citations in two CT-based case reports [1,2] we are not aware of previous descriptions of the CMR features of caseous calcification of the mitral annulus. We believe the hallmarks of this condition to be low signal in both T1-W and T2-W sequences, both before and after contrast, associated with a slightly-darker-than-myocardium signal in SSFP sequences. Further examples need to be studied,

Table I: CMR appearances found in the reported cases of caseous calcification of the mitral annulus

\begin{tabular}{|c|c|c|c|c|}
\hline Pre-contrast TI-W & Pre-contrast T2-W & Pre-contrast bSSFP & First pass perfusion & Delayed Enhancement \\
\hline DARK & BLACK & Slightly darker than myocardium & Not perfused & $\begin{array}{l}\text { Enhanced border surrounding a non-enhanced } \\
\text { core }\end{array}$ \\
\hline
\end{tabular}


but it may prove feasible to diagnose caseous calcification of the mitral annulus by CMR without the need for further CT examination.

\section{References}

I. Vanovermeire OM, Duerinckx AJ, Duncan DA, Russell WG: Caseous calcification of the mitral annulus imaged with 64slice multidetector $C T$ and magnetic resonance imaging. The International Journal of Cardiovascular Imaging 2006, 22:553-559.

2. Lubarsky L, Jelnin V, Marino N, Hecht HS: Images in cardiovascular medicine. Caseous calcification of the mitral annulus by 64-detector-row computed tomographic coronary angiography: a rare intracardiac mass. Circulation 2007, I I6(5): | I 4-5.

3. Harpaz D, Auerbach I, Vered Z, Motro M, Tobar A, Rosenblatt S Caseous calcification of the mitral annulus: a neglected, unrecognized diagnosis. J Am Soc Echocardiogr 200I, I 4:825-3I.

4. Marcu CB, Ghantous AE, Prokop EK: Caseous Calcification of the Mitral Valve Ring. Heart Lung and Circulation 2006, I 5:187-I88.

5. Fieno DS, Saouaf R, Thomson LE, Abidov A, Friedman JD, Berman DS: Cardiovascular magnetic resonance of primary tumors of the heart: a review. J Cardiovasc Magn Reson 2006, 8(6):839-53. 